\title{
A solar energy mix for a green and sustainable future
}

\begin{abstract}
The current work is present in a form of practical approach of solar energy systems which are part of the current energy mix. This paper intends to explain the importance of relying on solar energy generating power plants as an answer to climate changes and international agreement on pollution reduction. The current work focuses on the dispatchability issue, overcome by concentrated solar thermal power plants.
\end{abstract}

Volume 2 Issue 2 - 2018

\author{
Francesco Anglani, Selene Pennetta \\ Queensland University of Technology, Australia \\ Correspondence: Francesco Anglani, University Queensland \\ University of Technology (QUT), Address 2 George Street, \\ Brisbane, Australia, Tel +6/424803992, \\ Email Francesco.anglani@qut.edu.au, \\ Francesco.anglani@gmail.com
}

Received: October 15, 2017 | Published: November 15, 2018

\section{The intermittent issue}

The growth of solar energy generating systems starts in the 1970s with first applications of solar cell and photovoltaic (PV) technologies. The rapid decrease of manufacturing costs thanks to a massive growth in mass production of photovoltaic modules and subsequent help from government incentives that have facilitated the deployment of this technology in every sector. ${ }^{1}$ The introduction of renewable sources changed the background of the energy sector. The energy generated by biomass, geothermal, wind, photovoltaic (PV) and concentrated solar thermal (CST) plants has introduced indeed new challenges for energy operators who have had to address energy market needs differently. The United Nations Framework Convention on Climate Change (UNFCCC) and the Kyoto protocol have led to agreement between nations on climate change and the need to reduce greenhouse gas emissions. The mitigation of $\mathrm{CO}_{2}$ and $\mathrm{NOx}$ pushed energy operators to rely on variety of "green" energy production technologies. Thus, the development and installation of photovoltaics and wind plants widely increased during past three decades generating a learning curve for both manufacturers and energy market operators. The downside of both renewable technologies is the intermittence of electrical generation as it is function of weather conditions. A full conversion to solar and wind energy is not then a feasible solution to satisfy a flexible and continuous energy market demand, hence these technologies are usually employed to respond to energy peak requests, mostly.

Operators have to address the energy mix of primary sources such as coal, gas and oil coupled with renewable source from a technical and economic point of view. Since primary sources cannot be completely switched off because the energy requirements to be energy reactivated are enormous and costly, coal and gas power stations are thus set to a minimum threshold and coupled with alternative sources such as solar, wind and geothermal energy to respond to hourly market demand. Secondly, wind and photovoltaics energy production needs to be moderated to not overload the grid system fed mostly by standard power energy stations. Dispatchbility as solar energy moves towards a more mature technology, a decrease of component costs is observed, with improvements to the levelized cost of energy (LCOE). Coal and gas power stations are still prominent because of their mature technology, lower costs and high dispatchability. On the other hand, the research community has taken their side effects on global warming and pollution seriously, being willing to move towards different energy. Parallel to the development of photovoltaics, the promising concentrated solar thermal (CST) technology provided a solution to the intermittency issue of previous technologies discussed above. Unlike PV, CST technologies rely on a system of hot and "cold" tanks to stock heat energy able to satisfy power generation overnight. This technology needs to be at utility scale like coal or gas fire power stations and are not suited to rooftop solar. Concentrated solar thermal technology is the most proven and lowest cost largescale solar power technology available nowadays. ${ }^{2}$ CST plants can only use direct solar radiation, called beam radiation or Direct Normal Irradiance (DNI), the fraction of solar radiation which is not deviated by clouds, fumes or dust in the atmosphere and that reaches the Earth's surface as a normal beam. Appropriate site locations for CST plants are normally in arid to semi-arid regions, where the DNI resource is very high, acceptable production costs for commercial CSP plants are typically where DNI exceeds about $2000 \mathrm{kWh} / \mathrm{m}^{2}$ year. Parabolic trough collectors (PTCs), concentrates the direct normal insolation (DNI) along a tubular receiver (Figure 1) inside which the heat transfer fluid (HTF) flows. ${ }^{3}$ The fluid, generally oil, molten salts or simply water, collects the thermal energy and feeds a Rankine cycle turbine-generator that produce electrical energy (Figure 1). A storage can be integrated to enhance dispatchability (Figure 2), or hybridizing with natural gas allows this technology to be fully operative overnight to meet peak demand periods. PTC is the widest technology used in concentrated solar power since it has been extensively developed and tested with the installation Mojave desert (354MW) made up of nine Solar Energy Generating Systems (SEGS): SEGS I-II (44 MW) are located at Dagget; SEGS III-VII (150 MW) are installed at Kramer Junction; SEGS VIII-IX (160 MW) are placed at Harper Lake.

A more recent technology is the power tower that consists of several flat mirrors called heliostats which track the sun and reflect sun's rays to a receiver placed at the top of the tower, (Figure 3). In comparison with parabolic trough technology, tower systems have higher efficiency thanks to high temperature reached in the receiver (up to $565^{\circ} \mathrm{C}$ ) and less thermal losses. The thermal energy is captured by the heat transfer fluid flowing through the receiver and pumped to the steam generator. Even tower plants can be coupled with thermal 
storage able to feed the Rankine steam-turbine power block to meet the peak load requirements overnight. The Linear Fresnel technology is the discretization of the parabolic trough collectors in small flat ground-mounted mirrors which concentrated sun's rays onto the tube receiver fixed above the mirror facets (Figure 4). The optical efficiency is lower than troughs one, but it offers cost savings for less land required, tight mirrors spacing and ground location of mirrors' facets which require a less expensive manufacturing process. Finally, this technology is characterized by a field of parabolic dishes and each of them reflects sunlight onto a Sterling engine that produces electricity directly. These parabolic dishes track sunlight in two axes and are able to collect the maximum amount of DNI throughout the day achieving very high temperature (up to $800^{\circ} \mathrm{C}$ ) and high efficiency, converting over than $30 \%$ of sunlight to electrical energy (Figure 5).

CST power plants and coal generating power plants then, are

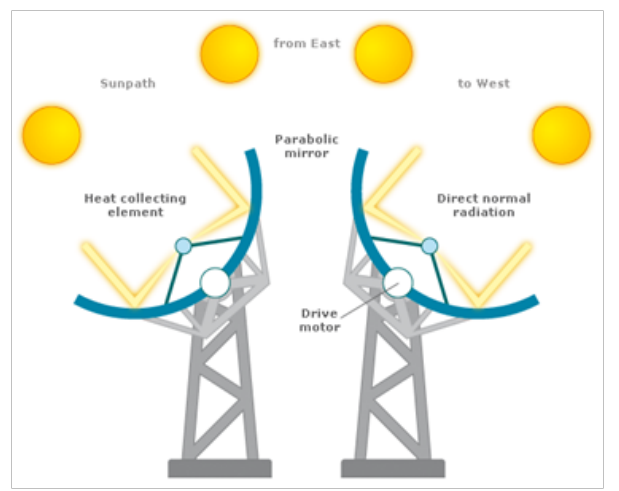

Figure I Parabolic Trough Collectors (PTCs) tracking solar radiations (DNI).

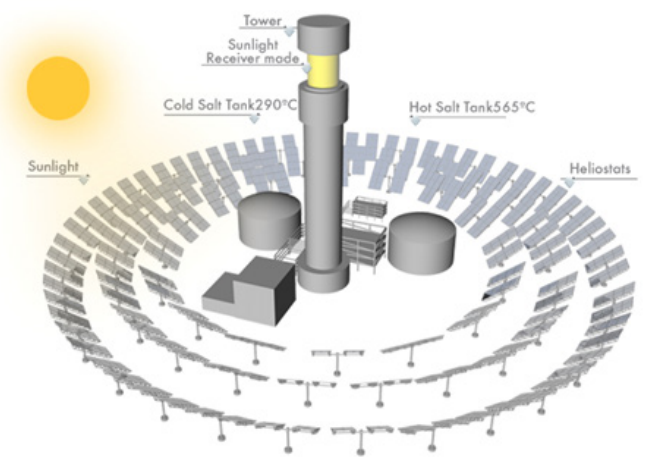

Figure 3 Power tower technology. similar since both are based upon Rankine cycle steam turbines that drive 3-phase generators. The difference is to be found on how CST plants produced heat energy compared to coal or gas. Instead of burning fuel to heat the boiler, CST technology uses a field of flat or curved mirrors also known as heliostats or parabolic trough mirrors to concentrate energy from the sun to heat boilers and provide pressurized steam to steam turbines. Usually conventional boilers do not require an intensive maintenance since its uptime depends on a simple combustion process and a normal maintenance activity is expected to guarantee the correct operation. CST boilers, on the other hand, are driven by the sun which is a variable heat source. The performance depends upon time of day and upon the performance of the collector mirrors that are used to concentrate the sun's energy. The performance of the system can be degraded significantly due to dust accumulation onto mirror surfaces that reduce collection efficiency via scatter and absorption of reflected light.

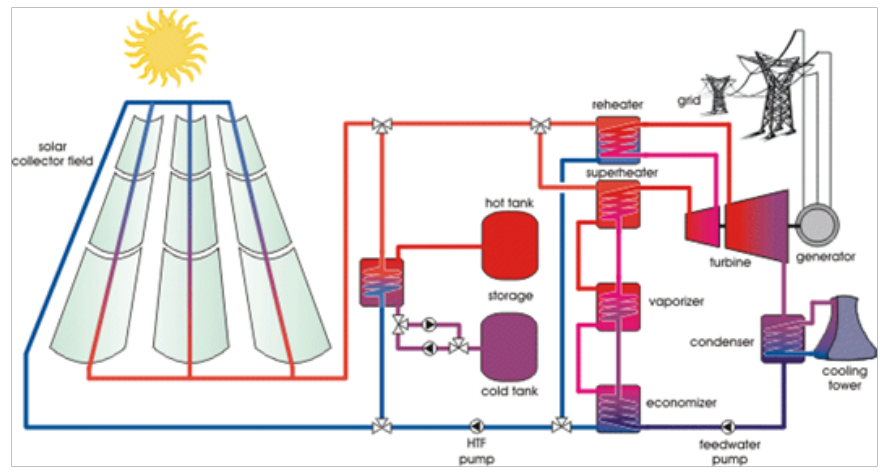

Figure 2 Parabolic trough system schematic.

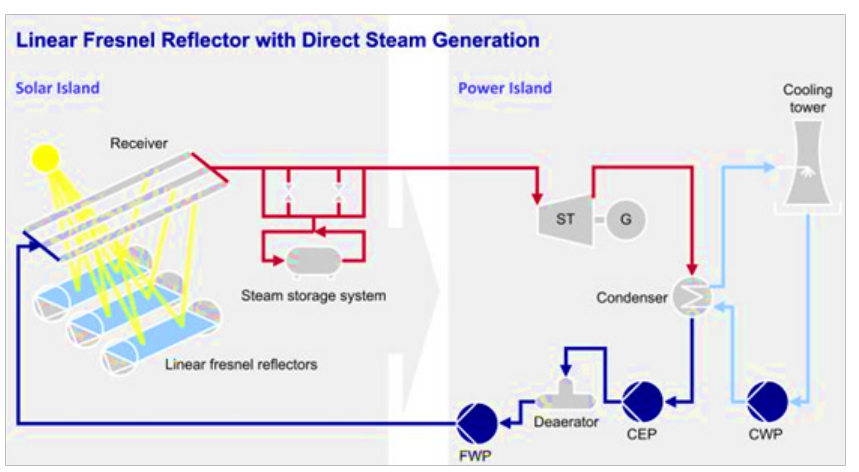

Figure 4 Linear Fresnel technology schematic.

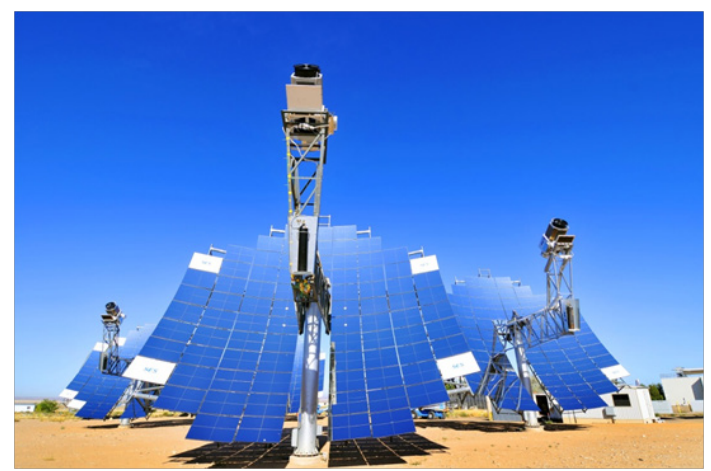

Figure 5 Parabolic-shaped dish collector with Stirling engine onto the receiver. 


\section{Conclusion}

An energy mix more balanced on green energy solution is definitely the more sustainable solution to address the current and future energy crisis with less environmental impact. The recovery and replacement of fuel based generating system in favour of more green energy sources along with the adoption of more sustainable lifestyle, represents a necessary solution to contain the global warming effect. Moreover, recent discoveries by the research community and the development of lithium ion battery storage systems for automotive, household and industrial provided s by Tesla, enhances the concept of living off grid to a next level. The electricity generated by photovoltaic and wind turbines can now be stored in battery packs and employed when needed. This technology combined with geothermal, biomass, and CST power plants represent a solid alternative energy mix able to replace fuel based power with a beneficial decrease of the pollution.

\section{Conflicts of interest}

Authors declare that there is no conflict of interest.

\section{References}

1. Costa SCS, Diniz A, Kazmerski LL. Dust and soiling issues and impacts relating to solar energy systems: Literature review Dust and soiling issues and impacts relating to solar energy systems: Literature review update for 2012-201. Renewable \& Sustainable Energy Reviews.ol. 2016;63:33-61.

2. Fernandez-Garcia A, Zarza E, Valenzuela L, et al. Parabolic-trough solar collectors and their applications. Renewable and Sustainable Energy Reviews. 2010;14(7):1695-1721.

3. USD Energy. Concentrating Solar Power Commercial Application Study: Reducing Water Consumption of Concentrating Solar Power Electricity Generation. 2007.

\section{Acknowledgments}

None. 